\title{
Solvent Effect on the Nature of the Metallamacrocycles Formed: Formation of Octanuclear and Dodecanuclear Manganese Metalladiazamacrocycles ${ }^{\dagger}$
}

\author{
Rohith P. John, Kyungjin Lee, Kyungjae Lee, Mira Park, and Myoung Soo Lah ${ }^{*}$ \\ Department of Chemistry and Applied Chemistry, College of Science and Technologv, Hanyang University, \\ Ansan, Gynuggi 426-791, Korea. E-mail: mslah@hamang.ackr
}

Received June 5, 2007

\begin{abstract}
Depending on the choice of coordinating solvent molecules, the size and nuclearity of metallamacrocycles formed could be controlled. When a manganese ion was assembled with a pentadentate ligand, $N$-trans(pentenoyl)salicylhydrazide in amide solvents such as dimethylformamide (DMF) or dimethylacetamide $(\mathrm{DM} \Lambda)$, an $S_{6}$ symmetry puckered dodecanuclear metalladiazamacrocycle with a $\cdots(\Lambda \Lambda \Lambda \Lambda)(\Lambda \Lambda \Lambda \Lambda) \cdots$ chiral sequence was obtained. Ilowever, in an alcoholic solvent such as methanol or ethanol while keeping the other conditions the same, an $S_{8}$ symmetry octanuclear metalladiazamacrocycle with a $\cdots(\Lambda \Delta)(\Lambda \Delta) \cdots$ chiral sequence was obtained.
\end{abstract}

Key Words : Self-assembly, Molecular polygon, Octanuclear metallamacrocycle, Dodecanuclear metallamacrocycle, Solvent effect

\section{Introduction}

The creation of metal-organic architectures such as molecular polygons, ${ }^{1}$ metal-organic polyhedra, ${ }^{2}$ and metalorganic frameworks ${ }^{3}$ have attracted much attention in recent years because of their potential applications in various fields, such as single molecular magnets, ${ }^{4}$ electroluminescence, ${ }^{5}$ storage, ${ }^{6}$ and catalysis. ${ }^{7}$ Because the functionalities of these materials are closely related to their structure, the rational design and control of architectures is of importance. Although metallamacrocycles - metal-organic polygons are among the simplest forms of metal-organic species, many difficulties still remain in the prediction of the final self-assembled product from metal and organic building blocks. During the last 10 years several metalladiazamacrocycles - diaza-bridged metallamacrocycles - having diverse nuclearities and sizes have been prepared via the cyclic linkage of ditopic pentadentate ligands, $\mathrm{N}$-acylsalicylhydrazide, and ditopic tricationic octahedral metal ions. ${ }^{8}$ Control of nuclearity and size of the metalladiazamacrocycles was achieved by careful control of the steric repulsion offered by the $N$-acyl terminal group of the ligand. When other factors such as choice of metal ion and identity of the ligand remain the same, the nature of the product formed is primarily the same. When the pentadentate ditopic ligands having linear $N$-acyl terminal groups are combined with octahedral manganese(III) ion, the solvent that serves as a monodentate ligand at the sixth coordination site of the macrocyclic ring metal center has no significant effect on the macrocyclic ring system. ${ }^{9}$ Regardless of the solvent molecules, the products formed are exclusively of hexanuclear manganese metallamacrocycles.

Here we demonstrate a simple example of a system where

"This paper is dedicated to professor Sang Chul Shim on the occasion of his honorable retirement. a change in solvent has made a significant difference to the nature of the product formed.

\section{Experimental Section}

\section{Ligand synthesis}

$\mathrm{N}$-2-Pentenoylsalicylhydrazide $\left(\mathrm{H}_{3}\right.$ tpeshz): The ligand was prepared following the literature method. ${ }^{10} 1.24 \mathrm{~mL}$ $(10.0 \mathrm{mmol})$ of trimethylacetyl chloride was added to $20 \mathrm{~mL}$ of chloroform at $0{ }^{\circ} \mathrm{C}$, containing $1.54 \mathrm{~mL}(11.0 \mathrm{mmol})$ of triethylamine and $1.03 \mathrm{~g}(10.0 \mathrm{mmol})$ of trans-2-pentenoic acid, while stirring. After stirring for about $20 \mathrm{~min}$, the solution was slowly brought to ambient temperature. Then $1.40 \mathrm{~g}(9.00 \mathrm{mmol})$ of salicylhydrazide was added and stirred over a period of $30 \mathrm{~min}$. When $10 \mathrm{~mL}$ hexane was added to the solution, a white precipitate began to appear. The white product was filtered out and washed with a 1:1 mixture of chloroform and hexane, and then with small portions of ether, followed by drying in vacuum. Yield: 1.50 g $\left(71.0 \%\right.$ ). Elemental analysis for $\mathrm{C}_{12} \mathrm{H}_{14} \mathrm{~N}_{2} \mathrm{O}_{3}$. Calc.: C $61.53, \mathrm{H} 6.02, \mathrm{~N} 11.96 \%$, found: C $61.85, \mathrm{H} 6.54, \mathrm{~N} 11.76 \%$. 'H NMR spectrum (300 MHz, dmso-d 6 , ppm): 11.89 (bs, $1 \mathrm{H},-\mathrm{NH}), 10.70(\mathrm{bs}, 1 \mathrm{H},-\mathrm{NH}), 10.40(\mathrm{~s}, 1 \mathrm{H},-\mathrm{OH}), 7.91(\mathrm{~d}$, $1 \mathrm{H}), 7.44(\mathrm{t}, 1 \mathrm{H}), 6.96(\mathrm{~d}, 1 \mathrm{H}), 6.94(\mathrm{t}, 1 \mathrm{H}), 6.84(\mathrm{td}, 1 \mathrm{H})$, $6.05(\mathrm{~d}, 1 \mathrm{H}), 2.20\left(\mathrm{q}, 2 \mathrm{H}, \mathrm{CH}_{2}\right), 1.02\left(\mathrm{t}, 3 \mathrm{H}, \mathrm{CH}_{3}\right) .{ }^{13} \mathrm{C} \mathrm{NMR}$ spectrum $\left(75 \mathrm{MHz}, \mathrm{dmso}-d_{\hbar}, \mathrm{ppm}\right): 166.20,163.52,158.82$, $146.36,134.07,128.62,120.65,119.16,117.32,114.82$, 24.65, 12.3. IR spectrum $\left(\mathrm{KBr}, \mathrm{cm}^{-1}\right): 3172(\mathrm{~m}), 3118(\mathrm{~m})$, $3067(\mathrm{~m}), 1673(\mathrm{w}), 1646(\mathrm{~m}), 1559(\mathrm{~s}), 1481(\mathrm{~s})$.

Preparation of metalladiazamacrocycles

[Mn (t) $_{12}$ (peshz) $)_{12}$ (DMA) $)_{12}$ ], 2: $0.049 \mathrm{~g}(0.21 \mathrm{mmol})$ of $\mathrm{H}_{3}$ tpeshz was dissolved in $10 \mathrm{~mL}$ of DMA and $0.052 \mathrm{~g}(0.21$ mmol) of solid $\mathrm{Mn}(\mathrm{OAc})_{2} \cdot 4 \mathrm{H}_{2} \mathrm{O}$ was added. Dark brown needle like crystals were harvested after $12 \mathrm{~d}$. Yield: $0.087 \mathrm{~g}$ $(79 \%)$. X-ray quality single crystals were obtained from a batch of $10 \mathrm{~mL}$ of 1:1 DMA solution of $-0.1 \mathrm{mmol}$ of 
manganese acetate and $\mathrm{H}_{3}$ tpeshz over a period of $24 \mathrm{~d}$. IR (KBr pellet, $\mathrm{cm}^{-1}$ ): 3436 (br, m) $2963(\mathrm{~m}), 2933(\mathrm{~m}), 1655$ (m), $1619(\mathrm{~m}), 1600(\mathrm{~s}), 1565(\mathrm{~s}), 1514(\mathrm{~s}), 1496(\mathrm{~s}), 1465$ (m), 1445 (m), 1407 (s), 1362 (s), $1324(\mathrm{~m}), 1247$ (m), 1147 (w), 1109 (w), 1033 (w), 969 (w), 919 (w), 862 (w), 795 (w), $754(\mathrm{~m}), 696(\mathrm{~m}), 686(\mathrm{~m}), 651(\mathrm{w}), 605(\mathrm{~m}), 554(\mathrm{w}), 480$ (w), 438 (w), 423 (w). Elemental data for [Mn ${ }_{12}(\text { tpeshz) })_{12}-$ (DMA) $\left.)_{10}\left(\mathrm{H}_{2} \mathrm{O}\right)_{2}\right]\left(\mathrm{Mn}_{12} \mathrm{C}_{184} \mathrm{H}_{22} \mathrm{~N}_{34} \mathrm{O}_{48}\right)$. Calc.: C $50.91, \mathrm{H}$ $5.25, \mathrm{~N} \mathrm{10.97 \%}$, found: C 50.74, H 5.19, N 11.15\%.

[Mns(tpeshz) $\left.)_{8}(\mathrm{MeOH})_{8}\right], 3: 0.094 \mathrm{~g}(0.40 \mathrm{mmol})$ of $\mathrm{H}_{3}$ tpeshz was dissolved in $10 \mathrm{~mL}$ of $\mathrm{MeOH}$ in a $15 \mathrm{~mL}$ vial and $0.098 \mathrm{~g}(0.40 \mathrm{mmol})$ of $\mathrm{Mn}(\mathrm{OAc})_{2} \cdot 4 \mathrm{H}_{2} \mathrm{O}$ was added to the solution. After standing for $15 \mathrm{~d}$, dark brown block crystals were obtained $(0.089 \mathrm{~g}, 71 \%$ yield). IR ( $\mathrm{KBr}$ pellet, $\mathrm{cm}^{-1}$ ): 3442 (br), 1653 (w), 1601 (m), 1567 (m), 1497 (s), 1445 (w), 1408 (s), 1363 (s), 1321 (w), 1239 (w), 860 (w), 760 (w), 687 (w). Elemental analysis for [Mns(tpeshz) $)_{8-}$ $\left.\left(\mathrm{H}_{2} \mathrm{O}\right)_{8}\right]\left(\mathrm{C}_{96} \mathrm{H}_{104} \mathrm{~N}_{16} \mathrm{O}_{32} \mathrm{Mn}_{8}\right)$. Calc.: $\mathrm{C} 47.38, \mathrm{H} 4.31, \mathrm{~N}$ $9.21 \%$, found: C $47.53, \mathrm{H} 4.15, \mathrm{~N} 9.27 \%$.

[Mns(tpeshz) $\left.)_{8}(\mathbf{E t O H})_{8}\right], 4: 0.094 \mathrm{~g}(0.40 \mathrm{mmol})$ of $\mathrm{H}_{3}$ tpeshz was dissolved in $10 \mathrm{~mL}$ of $\mathrm{EtOH}$ in a $15 \mathrm{~mL}$ vial and $0.098 \mathrm{~g}(0.40 \mathrm{mmol})$ of $\mathrm{Mn}(\mathrm{OAc})_{2} \cdot 4 \mathrm{H}_{2} \mathrm{O}$ was added to the solution. After standing for $15 \mathrm{~d}$, dark brown block crystals were obtained $(0.104 \mathrm{~g}, 79 \%$ yield). $\mathbb{R}$ ( $\mathrm{KBr}$ pellet, $\mathrm{cm}^{-1}$ ): 3444 (br), 1654 (w), 1601 (m), 1566 (m), 1495 (s), 1409 (s), 1365 (s), 1321 (w), $687(\mathrm{w})$. Elemental analysis for [Mns (tpeshz) $\left.)_{8}\left(\mathrm{H}_{2} \mathrm{O}\right)_{8}\right]\left(\mathrm{C}_{96} \mathrm{H}_{10} \mathrm{~N}_{16} \mathrm{O}_{32} \mathrm{Mnn}_{8}\right)$. Calc.: C 47.38, H 4.31, N 9.21\%, found: C 47.51, H 4.21, N 9.40\%.

Crystallographic data collections and refinements of structures. The diffraction data were measured at $173 \mathrm{~K}$ with Mo K $\alpha$ radiation ( $\lambda=0.71073 \AA$ ) on a Bruker SMART CCD equipped with a graphite crystal, incident-beam monochromator. The SMART and SAINT software packages" were used for data collection and integration, respectively. The collected data were corrected for absorbance using $\mathrm{SADABS}^{12}$ based upon Laue symmetry using equivalent reflections.

Crystal structure determination for $\left[\mathbf{M n}_{12}(t \text { peshz })_{12}-\right.$ (DMA) $\left.)_{12}\right] \cdot 3 \mathrm{DMA} \cdot \mathbf{4 \mathrm { H } _ { 2 }} \mathrm{O},\left\{2 \cdot 3 \mathrm{DMA} \cdot 4 \mathrm{H}_{2} \mathrm{O}\right\}, 2: \mathrm{Mn}_{12} \mathrm{C}_{224}-$ $\mathrm{H}_{266} \mathrm{~N}_{42} \mathrm{O}_{56}, \mathrm{fw}=5102.07 \mathrm{~g} \mathrm{~mol}^{-1}$, triclinic, space group $P \overline{1}$, $a=14.733(3), b=21.583(4), c=22.482(4) \AA, \alpha=$ $64.793(3)^{\circ}, \beta=76.553(3)^{\circ}, \gamma=82.154(3)^{\circ}, V=6284.8(19)$ $\AA^{3}, T=173(2) \mathrm{K}, \mathrm{Z}=1, \mu(\mathrm{Mo} \mathrm{K} \alpha, \lambda=0.71073 \AA)=0.662$ $\mathrm{mm}^{-1}, 34126$ reflections were collected, 23949 were unique $\left[\mathrm{R}_{\mathrm{int}}=0.0376\right]$. The structure was solved by direct methods and refined by block diagonalized matrix least-squares calculations with the SHELXTL-PLUS software package. ${ }^{13}$ A dodecanuclear metallamacrocycle was identified in a crystallographic inversion center. Of the six coordinated DMA molecules at the asymmetric unit, two are disordered. The asymmetric unit also contains five non-coordinating DMA sites (one fully occupied, four disordered and half occupied DMA sites) and four water sites. All non-hydrogen atoms except those of the disordered coordinating solvents and those of the non-coordinating structural solvent molecules were refined anisotropically; hydrogen atoms were assigned isotropic displacement coefficients $\mathrm{U}(\mathrm{H})=1.2 \mathrm{U}(\mathrm{C})$ or $1.5 \mathrm{U}\left(\mathrm{C}_{\text {methy }}\right)$, and their coordinates were allowed to ride on their respective atoms. The refinement of the structure converged at a final $R 1=0.0815, w R 2=0.2197$ for 15419 reflections with $I>2 \sigma(I)$.

Refinement details for $\left[\mathrm{Mn}_{12}(\text { tpeshz })_{12}(\mathrm{DMA})_{12}\right], 2$, after using the SQUEEZE routine of PLATON: ${ }^{14} \mathrm{Mn}_{12} \mathrm{C}_{192} \mathrm{H}_{240}$ $\mathrm{N}_{16} \mathrm{O}_{48}$, fw $=4479.48 \mathrm{gmol}^{-1}$, triclinic, space group $P \overline{1}, a=$ $14.733(3), b=21.583(4), c=22.482(4) \AA, \alpha=64.793(3)^{\circ}, \beta$ $=76.553(3)^{\circ}, \gamma=82.154(3)^{\circ}, V=6284.8(19) \AA^{3}, T=173(2)$ $\mathrm{K}, \mathrm{Z}=1, \mu(\mathrm{Mo} \mathrm{K} \alpha, \lambda=0.71073 \AA)=0.650 \mathrm{~mm}^{-1}, 34119$ reflections were collected, 23946 were unique $\left[R_{\text {int }}=0.0364\right]$. The structure refinement was further performed after modification of the data for the non-coordinate lattice solvent molecules ( $1212.4 \AA^{3}, 19.3 \%$ of the crystal volume) with the SQUEEZE routine of PLATON, which led to better refinement and data convergence. Refinement of the structure converged at a final $R 1=0.0778, w R 2=0.2186$ for 23946 reflections with $I>2 \sigma(I), R 1=0.1130, w R 2=0.2372, \mathrm{GOF}$ $=1.049$ for all 15121 reflections. The largest differences for peak and hole were 1.752 and $-0.969 \mathrm{e} \cdot \AA^{-3}$, respectively. A summary of the crystal and intensity data is given in Table S1.

Crystal structure determination for [Mns(tpeshz)s(MeOH) $)_{8}$-8.25MeOH, \{3+8.25MeOH\}, 3: $\mathrm{Mn}_{8} \mathrm{C}_{112.25} \mathrm{H}_{149-}$ $\mathrm{N}_{16} \mathrm{O}_{40.25}, \mathrm{fw}=2805.99 \mathrm{gmol}^{-1}$, monoclinic, space group $P 2{ }_{1} / n, a=14.469(2), b=14.704(2), c=62.866(9) \AA, \beta=$ $92.268(3)^{\circ}, V=13365(3) \AA^{3}, T=173(2) \mathrm{K}, Z=4, \mu(\mathrm{Mo}$ $\mathrm{K} \alpha, \lambda=0.71073 \AA)=0.814 \mathrm{~mm}^{-1}, 79263$ reflections were collected, 31599 were unique $\left[R_{\text {int }}=0.0957\right]$. The structure was solved by direct methods and refined by block diagonalized matrix least-squares calculations with the SHELXTLPLUS software package. ${ }^{13}$ An octanuclear metallamacrocycle and at least nine noncoordinating methanol solvent sites were identified as an asymmetric unit. All nonhydrogen atoms except those of the disordered coordinating solvent molecules and one noncoordinating structural solvent molecule were refined anisotropically; hydrogen atoms were assigned isotropic displacement coefficients $\mathrm{U}(\mathrm{H})=1.2 \mathrm{U}(\mathrm{C})$ or $1.5 \mathrm{U}\left(\mathrm{C}_{\text {mithyl }}\right)$, and their coordinates were allowed to ride on their respective atoms. The solvent coordination sites of the manganese ions are occupied by methanol molecules and one of them was disordered. The refinement converged to a final $R 1=0.1168$, and $w R 2=0.2276$ for 16689 reflections with $I>2 \sigma(I)$.

Refinement details for $\left[\mathrm{Mn}_{8}(\text { tpeshz })_{8}(\mathrm{MeOH})_{8}\right], 3$, after the SQUEEZE routine of PLATON: ${ }^{14} \mathrm{Mn}_{8} \mathrm{C}_{104} \mathrm{H}_{119} \mathrm{~N}_{16} \mathrm{O}_{32}$, $\mathrm{fw}=2544.67 \mathrm{gmol}^{-1}$, monoclinic, space group $P 2_{\mathrm{l}} / n, a=$ $14.469(2), b=14.704(2), c=62.866(9) \AA, \beta=92.268(3)^{\circ}, V$ $=13365(3) \AA^{3}, T=173(2) \mathrm{K}, \mathrm{Z}=4, \mu(\mathrm{Mo} \mathrm{K} \alpha, \lambda=0.71073$ $\AA)=0.802 \mathrm{~mm}^{-1}, 79263$ reflections were collected, 31599 were unique $\left[\mathrm{R}_{\mathrm{int}}=0.0843\right]$. The structure refinement was further performed after modification of the data for the noncoordinate lattice solvent molecules $\left(2114.9 \AA^{3}, 15.8 \%\right.$ of the crystal volume) with the SQUEEZE routine of PLATON, which led to better refinement and data convergence. Refinement of the structure converged at a final $R 1=$ $0.1035, w R 2=0.2694$ for 15812 reflections with $I>2 \sigma(I)$, $R 1=0.1750, w R 2=0.2961, \mathrm{GOF}=1.070$ for all 79263 
reflections. The largest differences for peak and hole were 0.935 and $-1.463 \mathrm{e} \cdot \AA^{-3}$, respectively. A summary of the crystal and intensity data is given in Table S2.

Crystal structure determination for [Mng(tpeshz) (EtOH) $\left.)_{8}\right] \cdot 8 \mathrm{EtOH} \cdot 1.5 \mathrm{H}_{2} \mathrm{O},\left\{4 \cdot 8 \mathrm{EtOH} \cdot 1.5 \mathrm{H}_{2} \mathrm{O}\right\}, 4: \mathrm{Mn}_{8-}$ $\mathrm{C}_{128} \mathrm{H}_{187} \mathrm{~N}_{16} \mathrm{O}_{41.50}, \mathrm{fw}=3053.46 \mathrm{gmol}^{-1}$, triclinic, space group $P \overline{1}, a=15.469(3), b=19.081(4), c=28.040(6) \AA, \alpha$ $=109.756(4)^{\circ}, \beta=94.681(4)^{\circ}, \gamma=102.418(4)^{\circ}, V=7499(3)$ $\AA^{3}, T=173(2) \mathrm{K}, \mathrm{Z}=2, \mu(\mathrm{Mo} \mathrm{K} \alpha, \lambda=0.71073 \AA)=0.731$ $\mathrm{mm}^{-1}, 30133$ reflections were collected, 20166 were unique $\left[\mathrm{R}_{\mathrm{int}}=0.0437\right]$. The structure was solved by direct methods and refined by block diagonalized matrix least-squares calculations with the SHELXTL-PLUS software package. ${ }^{13}$ An octanuclear metallamacrocycle and at least 12 noncoordinating structural solvent sites were identified as an asymmetric unit. All nonhydrogen atoms were refined anisotropically; hydrogen atoms were assigned isotropic displacement coefficients $\mathrm{U}(\mathrm{H})=1.2 \mathrm{U}(\mathrm{C})$ or $1.5 \mathrm{U}\left(\mathrm{C}_{\text {nethyl }}\right)$, and their coordinates were allowed to ride on their respective atoms. Two coordinating solvents and four noncoordinating solvents of bad geometry were refined with geometry restraints during the least-squares refinement. The solvent coordination sites of the manganese ions are occupied by ethanol molecules. In addition, eight ethanol molecules and one and a half water molecules per asymmetric unit were observed in the lattice. The refinement of the structure converged at a final $R 1=0.1103, w R 2=0.2548$ for 15356 reflections with $I>2 \sigma(h)$.

Refinement details for $\left.\left[\mathrm{Mn}_{8} \text { (tpeshz }\right)_{8}(\mathrm{EtOH})_{8}\right], 4$, after the SQUEEZE routine of PLATON: $:^{14} \mathrm{Mn}_{8} \mathrm{C}_{112} \mathrm{H}_{128} \mathrm{~N}_{16} \mathrm{O}_{32}$, fw $=$ $2649.82 \mathrm{gmol}^{-1}$, triclinic, space group $P \mathrm{~T}_{\mathrm{a}} a=15.469(3), b=$ $19.081(4), c=28.040(6) \AA, \alpha=109.756(4)^{\circ}, \beta=94.681(4)^{\circ}$, $\gamma=102.418(4)^{\circ}, V=7499(3) A^{3}, T=173(2) \mathrm{K}, \mathrm{Z}=2, \mu(\mathrm{Mo}$ $\mathrm{K} \alpha, \lambda=0.71073 \AA)=0.717 \mathrm{~mm}^{-1}, 30133$ reflections were collected, 20166 were unique $\left[\mathrm{R}_{\mathrm{in1}}=0.0427\right]$. The structure refinement was further performed after modification of the data for the non-coordinate lattice solvent molecules (1655.4 $\AA^{3}, 22.1 \%$ of the crystal volume) with the SQUEEZE routine of PLATON, which led to better refinement and data convergence. Refinement of the structure converged at a final $R \mathrm{I}=0.0971, w R 2=0.2463$ for 14799 reflections with $I$ $>2 \sigma(I), R 1=0.1174, w R 2=0.2559, \mathrm{GOF}=1.066$ for all 30133 reflections. The largest differences for peak and hole were 1.847 and $-0.843 \mathrm{e} \cdot \AA^{-3}$, respectively. A summary of the crystal and intensity data is given in Table S3.

Crystallographic data for the structures reported here have been deposited with Cambridge Crystallographic Data Center (Deposition No. CCDC 649288-649290). The data can be obtained free of charge via http://www.ccdc.cam. ac.uk/perl/catreg.cgi (or from the CCDC, 12 Union Road, Cambridge CB2 1EZ, UK; fax: +44 1223 336033; e-mail: deposit@ccdc.cam.ac.uk).

\section{Results and Discussion}

Previously, we have reported formation of a manganese dodecanuclear metallamacrocycle from a trianionic penta-
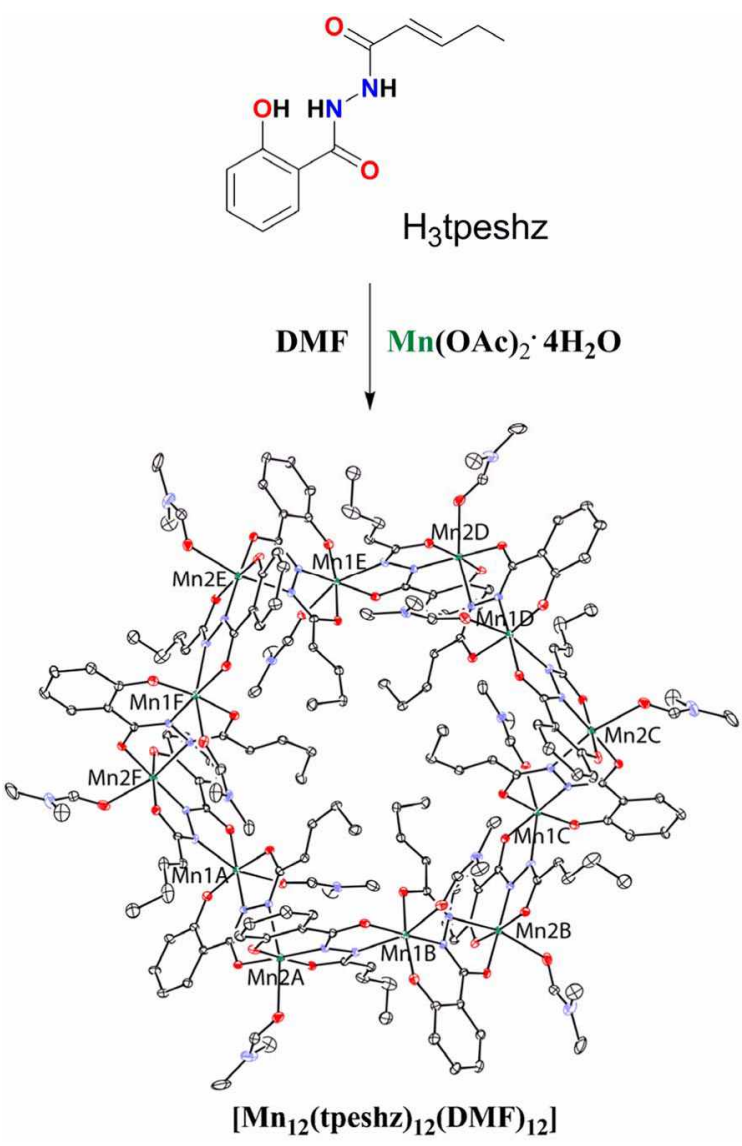

Scheme 1. Self-asscubly of an $S_{6}$ symmetry dodecanuclear manganese metalladiazamacrocycle from manganese ion and $\mathrm{H}_{3}$ ipeshz as a ditopic metal ion and a ditopic linker ligand, respectively, and DMF as a coordinating solvent.

dentate ligand - $N$-trans-(2-pentenoyl)salicylhydrazide $\left(\mathrm{H}_{3}\right.$ tpeshz) - in dimethylformamide (DMF) solution (Scheme 1). ${ }^{10}$

A trans double bond between the $\mathrm{C} \alpha$ and $\mathrm{C} \beta$ carbon atoms of the $\mathrm{N}$-acyl side chain of the ligand introduces a directional rigidity at the $N$-terminal position, and the steric repulsion between the $N$-acyl chains leads to an extended

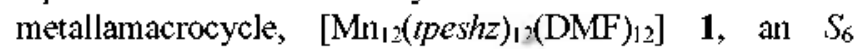
symmetry 36-membered dodecanuclear system, in which the metal centers are in the $(\Lambda \Lambda \Lambda)(\Lambda \Lambda \Lambda)$ chiral sequence. Six out of the $12 \mathrm{~N}$-terminal groups are directed towards the center of the macrocycle, and the remaining terminal groups are directed away from the macrocyclic ring. The chiral sequence and the consequent arrangement of the ligands lead to a more puckered ring conformation of the metalladiazamacrocycle than that of macrocycles in the $-(\Lambda \Delta)(\Lambda \Delta)$ - chiral sequence.

When the ligand is assembled with manganese as the metal ion in a similar solvent, dimethylacetamide (DMA), the same $S_{6}$ symmetry dodecanuclear metallamacrocycle, $\left[\mathrm{Mn}_{12}(\text { tpeshz })_{12}(\mathrm{DMA})_{12}\right] 2$, in a $(\Lambda \Lambda \Lambda \Delta)(\Lambda \Lambda \Lambda \Delta)$ chiral sequence is obtained (Figure 1). The only difference is a replacement of the sixth coordination site on the ring manganese ion, DMF in 1 with DMA in 2. 


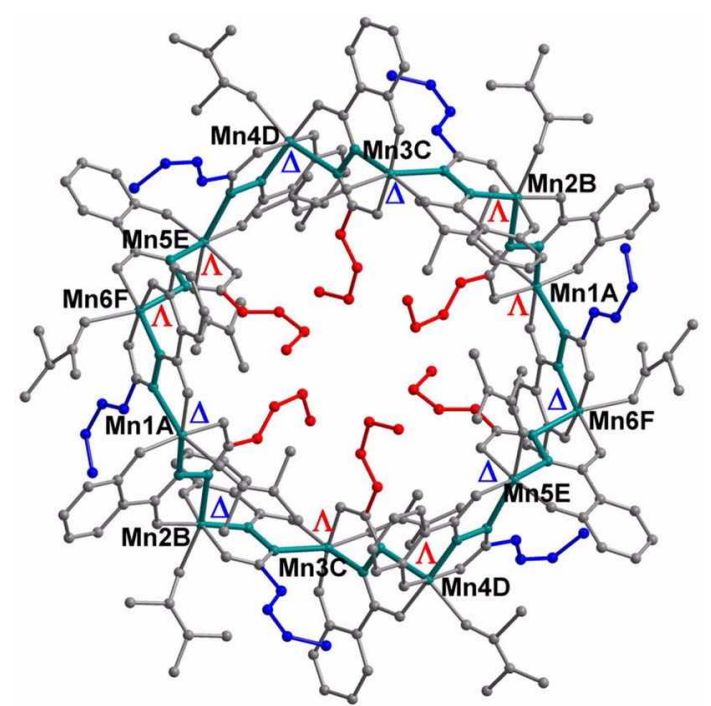

Figure 1. An ORTLP drawing of the $S_{6}$ symmetry puckered dodecanuclear metalladiazamacrocycle [Mn $\mathrm{Mn}_{\lfloor 2}(t p e s h z)_{1}$ (DMA) $\left.{ }_{\text {L2 }}\right], 2$.
However, a similar reaction in alcoholic solvents led to a quite different result, the formation of 24-membered $S_{8}$ symmetry octanuclear manganese metalladiazamacrocycles, in which the metal centers are in a $-(\Lambda \Delta)(\Lambda \Delta)$ - chiral sequence. When the ligand was allowed to self-assemble in a 1:1 ratio with manganese acetate as the metal source from methanol solution, dark-colored needle-like crystals were obtained from the deep brown solution in one day. The single crystal data showed the compound to be an octanuclear metallamacrocycle of [Mns(tpeshz $\left.)_{8}(\mathrm{MeOH})_{8}\right] \mathbf{3}$, with the ligand acting as a bridge and the metal ion as a connecting node (Figure 2). Each ligand binds to a metal ion as a chelating tridentate mode on one side and a chelating bidentate mode on the other side, thus serving as a ditopic linker between two metal ions. Eight cyclic repeats of the bridging mode generate the 24-membered octanuclear metallamacrocycle, where the chirality on the metal center originated from the tridentic and bidentic chelation modes on the octanuclear system altenlating $\Delta$ and $\Delta$ configu-
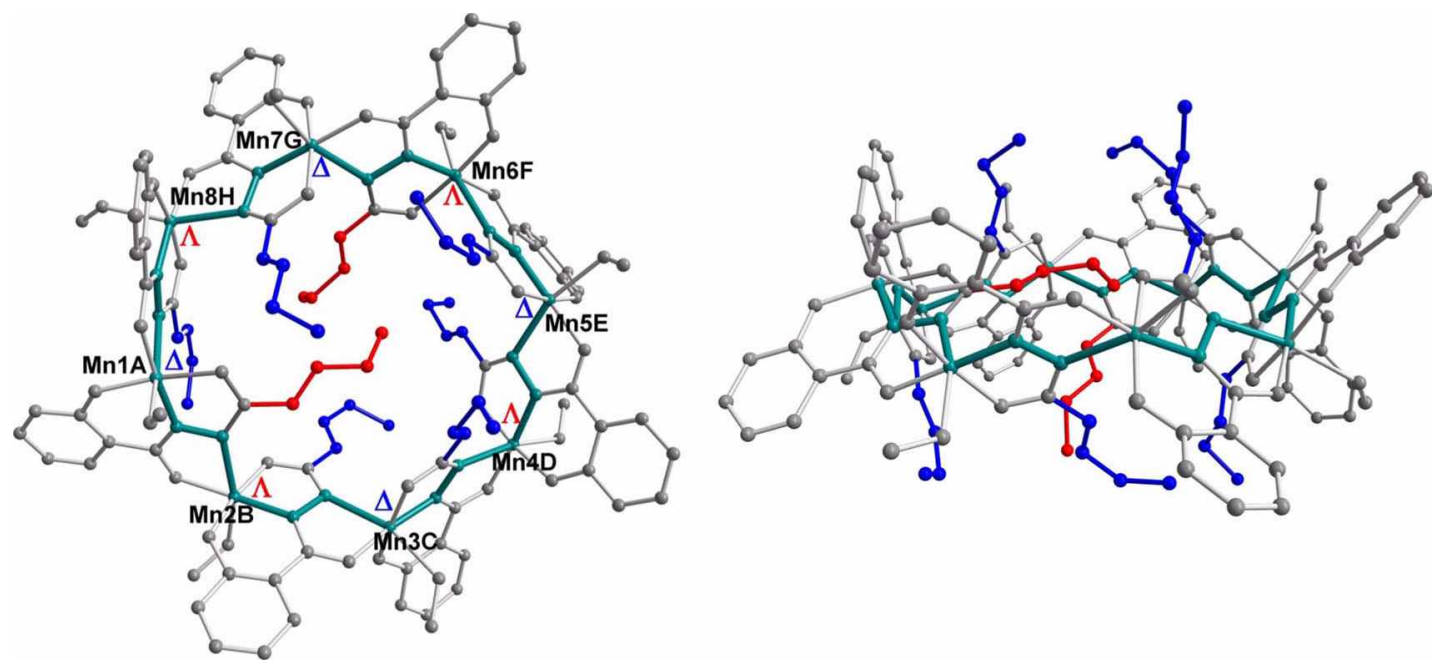

Figure 2. ORTEP drawings of the $S_{8}$ symmetry octanuclear metalladiazamacrocycle $\left[\mathrm{Mn}_{8}(t p e s h z)_{8}(\mathrm{MeOH})_{8}\right]$, 3. The $t$ rans-pentenoyl tails in and around the core of the macrocycle are represented in red and the remaining tails in blue. (a) Top view. (b) Side view.
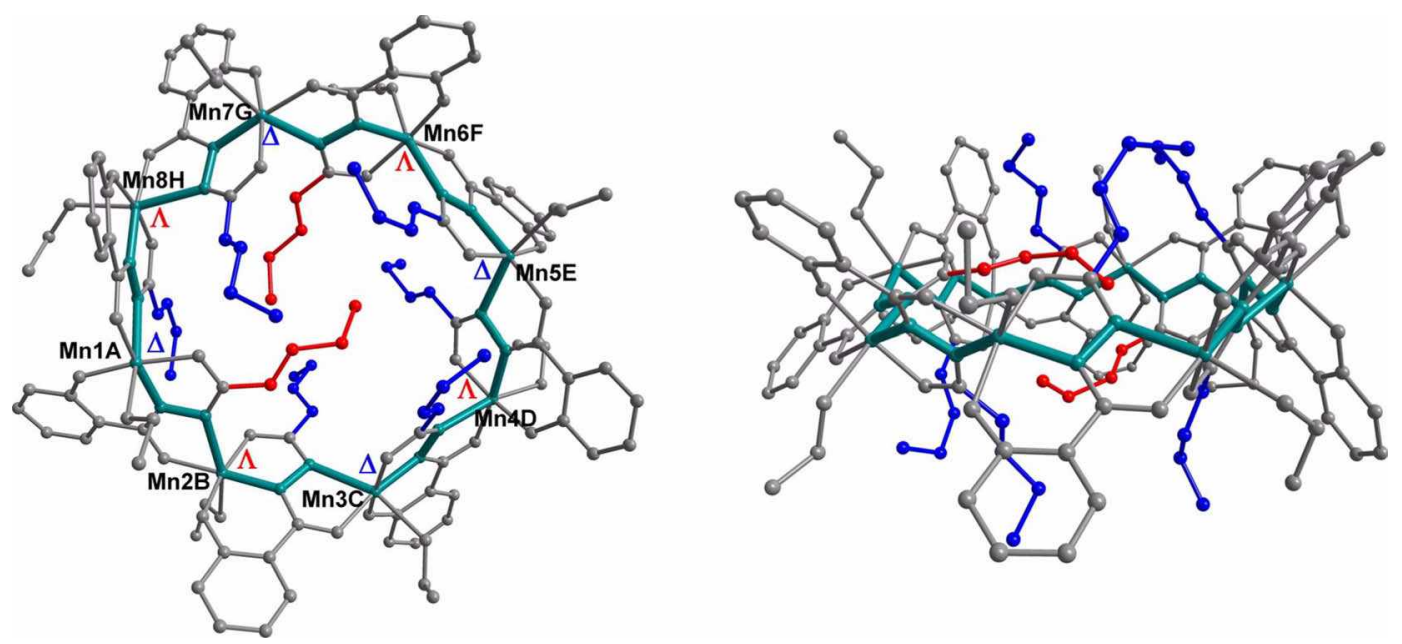

Figure 3. ORTEP drawings of the $S_{8}$ symınetry octanuclear metalladiazanacrocycle [Mnz(tpeshz) $($ EtoH) $]$, 4. The trans-pentenoyl tails in and around the core of the macrocycle are represented in red and the remaining tails in blue. (a) Top view. (b) Side view. 
rations. A similar reaction in ethanol solution also gives an isostructural octanuclear manganese metallamacrocycle, $\left[\mathrm{Mn}_{8}(t p e s h z)_{8}\left(\mathrm{EtOH}_{8}\right]_{4}\right.$, but with the difference that an ethanol satisfies the sixth coordination site instead of the methanol (Figure 3). The methanol-coordinated octanuclear metallamacrocycle, 3, and ethanol-coordinated octanuclear metallamacrocycle, 4 , have a similar arrangement of ligand units around the metal ions, while their local geometries vary only marginally.

The occurrence of $S_{8}$ symmetry octanuclear metallamacrocycles in a $-(M)(M)$ - chiral sequence in alcoholic solvents is unexpected because the macrocyclic ring strain in the less puckered $S_{8}$ symmetry metallamacrocycles is expected to be larger than that in the more puckered $S_{6}$ symmetry dodecanuclear metallamacrocycles. Because the hexanuclear system is the more preferred structure in the absence of significant steric influence from any of the ligand parts, the geometry around the metal center in a hexanuclear system was considered as the least strained. Therefore, the deviation in the geometry around the ring metal center and the $\mathrm{Mr}$ $\mathrm{Mn}-\mathrm{Mn}$ angles of the octanuclear or dodecanuclear systems from those of the most stable $S_{6}$ symmetry hexanuclear metallamacrocycles was assumed to reflect the extent of the macrocyclic ring strain. As shown in Table 1, the average Mn-Mn-Mn angles in the $S_{8}$ symmetry octanuclear metallamacrocycles 3 and 4 are $128.3^{\circ}$ and $127.5^{\circ}$, respectively, hence the deviation from those of the $S_{6}$ symmetry hexanuclear metallamacrocycles is approximately $15.2^{\circ}$. These values are much higher than those in $S_{6}$ symmetry puckered dodecanuclear metallamacrocycles 1 and $2\left(117.7^{\circ}, 119.8^{\circ}\right.$ for the average $\mathrm{Mn}-\mathrm{Mn}-\mathrm{Mn}$ angles and $6.1^{\circ}$ for the approximate deviation). Based on the extent of deviation from the values of the $\mathrm{Mn}-\mathrm{Mn}-\mathrm{Mn}$ angles in the hexanuclear systems, ${ }^{8 b, 8 c}$ we suggest that the ring strains in $\mathbf{3 / 4}$ are larger than those in $1 / 2$, hence metallamacrocycles 1 and 2 are more stable than metallamacrocycles 3 and 4 . To rationalize the formation of the more strained metallamacrocycles $3 / 4$ in alcoholic solvents, we analyzed the local geometry around the Jahn-Teller distorted manganese(III) centers because different extents of Jahn-Teller elongation might change the ring strain around the metal center and hence make the octanuclear system stable enough. However, a comparison of the Jahn-Teller distorted Mn-O4 and Mn-N2 bond lengths suggests that these minor variations in bond lengths cannot be considered as a major modulating factor of the ring strain, and the larger distortions of the related N1-Mn-N2 bond angles in the octanuclear systems compared with that of the puckered dodecanuclear systems do not support the reduction of the ring strain and the formation of the octanuclear metalladiazamacrocycles in alcoholic solvents (Table 1).

Although the formation of the $S_{8}$ symmetry octanuclear metallamacrocycles in alcoholic solvents carnot be fully rationalized, the extent of hydrophobic interaction may play an important role in the nature of the final product. In the case of $S_{8}$ symmetry octanuclear metallamacrocycles, all eight $N$-acyl tails are involved in the hydrophobic interaction around the cavity while only half of the $12 \mathrm{~N}$-acyl tails are involved in the hydrophobic interaction at the core of the $S_{6}$ symmetry dodecanuclear metallamacrocycles. In addition, one hydrophobic ethyl residue in metallamacrocycle 3 and two ethyl residues in 4 bend inwards to ensure better hydrophobic contact and for the optimum utilization of the available cavity at the center. All the remaining $N$-acyl tails are involved in hydrophobic interactions with each other. The existence of extensive hydrophobic interactions in the octanuclear systems might be a factor that stabilizes the octanuclear metallamacrocycles even in a more strained ring conformation. In a strict sense, the involvement of one or two $N$-acyl tails in the cavity for utilization of the hydrophobic interaction lowers the symmetry of the octanuclear system from $S_{8}$ to $C_{1}$. The two $N$-acyl tails, either both (Figure 2) or one towards the inner cavity and the other

Table 1. Comparison of the bond lengths $(\AA)$ and angles $\left({ }^{\circ}\right)$ around the ring metal center, and the geometries related to the ring conformation for various nuclearities of metalladiazamacrocycles

\begin{tabular}{|c|c|c|c|c|c|c|}
\hline & \multicolumn{2}{|c|}{$\begin{array}{l}S_{6} \text { syminetry hexanuclear } \\
\text { metallamacrocycles }\end{array}$} & \multicolumn{2}{|c|}{$\begin{array}{l}S_{6} \text { symmetry dodecanuclear } \\
\text { metallainacrocycles }\end{array}$} & \multicolumn{2}{|c|}{$\begin{array}{c}S_{\&} \text { symmetry octanuclear } \\
\text { metallainacrocycles }\end{array}$} \\
\hline & $\mathbf{a}^{4 t}$ & $\mathbf{b}^{\mathrm{b}}$ & 1 & 2 & 3 & 4 \\
\hline $\mathrm{Mn}-04$ & 2.175 & 2.247 & 2.248 & 2.224 & 2.243 & 2.237 \\
\hline $\mathrm{Mn}-\mathrm{N} 2$ & 2.262 & 2.310 & 2.288 & 2.274 & 2.246 & 2.238 \\
\hline N2-Mn-N1 & 102.4 & 101.9 & 101.9 & 104.1 & 107.6 & 107.6 \\
\hline N2-Mn-O3 & 88.9 & 90.4 & 86.7 & 89.7 & 92.2 & 91.8 \\
\hline Mn-Mn & 4.895 & 4.942 & 4.906 & 4.904 & 4.868 & 4.855 \\
\hline Mn-Mn-Mn & 112.0 & 113.3 & 117.7 & 119.8 & 128.3 & 127.4 \\
\hline
\end{tabular}

\begin{tabular}{ccccccccc}
\hline \multicolumn{7}{c}{ the dihedral angles between the ligand planes $v$. the best plane of the octahedral metallamacrocycles } \\
\hline & ligand A & ligand B & ligand C & ligand D & ligand E & ligand F & ligand G & ligand H \\
\hline $\mathbf{3}$ & $\mathbf{3 5 . 8}$ & 52.7 & 49.1 & 55.0 & $\mathbf{5 8 . 5}$ & $\mathbf{4 4 . 5}$ & $\mathbf{5 1 . 2}$ & $\mathbf{7 2 . 1}$ \\
$\mathbf{4}$ & $\mathbf{3 4 . 2}$ & 50.4 & 51.6 & 56.2 & $\mathbf{5 7 . 5}$ & $\mathbf{4 6 . 7}$ & $\mathbf{5 0 . 2}$ & $\mathbf{7 3 . 9}$ \\
\hline
\end{tabular}

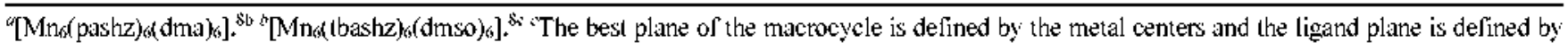
$\mathrm{O} 2, \mathrm{C} 7, \mathrm{N1}, \mathrm{N} 2, \mathrm{C8}$, and $\mathrm{O} 3$ atoms of each ligand. 
towards the cavity exterior (Figure 3), are located near the approximately central $S_{8}$ axis of the octanuclear metallamacrocycle. This distorts the overall ring conformations, and some ligands are located at a different orientation relative to the best macrocyclic ring planes (Figures 2 and 3 , Table 1).

\section{Conclusion}

We have prepared metallamacrocycles of two different nuclearities, a more puckered $S_{6}$ symmetry dodecanuclear manganese metalladiazamacrocycles and two less puckered $S_{8}$ symmetry octanuclear manganese metalladiazamacrocycles, by exploiting the difference in coordinating solvents while the rest of the parameters were kept the same. Even though the less puckered octanuclear systems have more ring strain than the more puckered dodecanuclear systems, the instability caused by the ring strain might have been alleviated by exploiting the more extensive hydrophobic interaction between the $\mathrm{N}$-acyl chains in and around the core of the macrocycle. Although it cannot be fully explained, the subtle differences in hydrophobicity and/or donor ability of the solvents used might be playing an important role in determining the nature of the final assembled metalladiazamacrocycles.

Acknowledgment. We gratefully acknowledge the financial assistance offered by Hanyang University (2006).

Supporting Information Available: The supporting material is available at bkcs website (www.kcsnet.orkt/bkes).

\section{References}

1. (a) Leininger, S.; Olenyuk, B.; Stang, P. J. Chem, Rev: 2000, 100 , 853. (b) Moulton, B.; Zaworotko, M. J. Chem. Rev, 2001, 101, 1629. (c) Holliday, B. J.; Mirkin, C. A. Angev. Chem, Int. Ed. Engl. 2001, 40, 2022. (d) Bodwin, J. J.; Culland, A. D.; Malkani, R. G.; Pecoraro, V. L. Coord. Chent. Rev: 2001, 216-217, 489. (e) Severin, K. Chem. Conmitim. 2006, 3859.

2. (a) Fujita, M.; Tominaga, M.; Hori, A.; Therrien, B, Acc. Chem. Res. 2005, 38, 37I. (b) Kubota, Y,; Biradha, K.; Fujita, M.; Sakamoto, S.; Yamaguchi, K, Bull. Chem. Soc. Jpn. 2002, 75, 559. (c) Caulder, D. L.; Raymond, K. N. J. Chem. Soc., Dalton Trans. 1999, 1185. (d) Dong. V. M.; Fiedler, D.; Carl, B.; Bergman, R. G.; Raymond, K. N. J. Ant. Chem. Soc. 2006, 128 , 14464. (e) Fiedler, D.; Bergman, R. G.; Raymond, K. N. Angew: Chem. Int. Ed. Engl. 2006, 45, 745. (f) Kawano, M.; Kobayashi, Y.; Ozeki, T.; Fujita, M. J. Am. Chem. Soc. 2006, 128, 6558. (g) Yoshizawa, M.; Kusukawa, T.; Fujila, M.; Yamaguchi, K. J. Am. Chem. Soc. 2000, 122, 6311. (h) Yoshizawa, M.; Miyagi, S.; Kawano, M.; Ishiguro, K.; Fujita, M. J. Am. Chem. Soc. 2004, 126, 9172. (i) Yoshizawa, M.; Takeyama, Y.; Okano, T.; Fujita, M. J. Am. Chem. Soc, 2003,125, 3243. (j) Tominaga, M.; Suzuki, K.; Kawano, M.; Kusukawa, T,; Ozeki, T,; Sakamoto, S.; Yamaguchi, K.; Fujila, M. Angew: Chem. Int. Ed, Engl. 2004, 43, 562l. (k) Yoshizawa, M.; Nakagawa, J.; Kumazawa, K.; Nagao, M.; Kawano, M.; Ozeki, T.; Fujita, M. Angen. Chem. Int. Ed. Engl. $2005,44,1810$.

3. (a) Eddaoudi, M.; Moler, D. B.; Li, H.; Chen, B.; Reineke, T. M.; O'Keeffe, M.; Yaghi, O. M. Acc. Chem. Res. 2001, 34, 319. (b)
Collon, F. A.; Lin, C.; Murillo, C. A. Acc. Chem, Res, 2001, 34, 759. (c) Yaghi, O. M.; O'Keeffe, M.; Ockwig, N. W.; Chae, H. K.; Eddaoudi, M.; Kim, J. Nature 2003, 423, 705. (d) Chae, H. K.; Siberio-Pérez, D. Y.; Kim, J.; Go, Y.; Eddaoudi, M.; Matzger, A.; O'Keeffe, M.; Yaghi, O. M. Natme 2004, 427, 523. (e) Li, H.; Laine, A.; O'Keeffe, M.; Yaghi, O. M. Science 1999, $283,1145$. (I) Kitagawa, S.; Kitaura, R.; Noro, S.-I. Angew: Chem, Int. Ed. Engl. 2004, 43, 2334 and references therein.

4. (a) Glaser, T.; Heidemeier, M.; Weyhermüller, T.; Hoffmann, R.D.; Rupp, H.; Müller, P. Angew: Chem. Int. Ed. Engl. 2006, 45, 6033. (b) Miyasaka, H.; Nakala, K.; Lecren, L.; Coulon, C.; Nakazawa, Y,; Fujisaki, T.; Sugiura, K.-I.; Yamashita, M.; Clérac, R. J. Am. Chem, Soc. 2006, J28, 3770. (c) Dendrinou-Samara, C.; Alexiou, M.; Zaleski, C. M.; Kampf, J. W. Kirk, M. L.; Kessissoglou, D. P.; Pecoraro, V. L. Angen. Chem. Int. Ed. Engl. 2003, 42, 3763. (d) Bell, A.; Aromi, G.; Teat, S. J.; Wernsdorfer, W.; Winpenny, R. E. P. Chem, Commn. 2005, 2808. (e) Hill, S.: Edwards, R. S.; Aliaga-Alcalde, N.; Christou, G Science 2003, 302, 1015. (I) Stamalalos, T. C.; Foguet-Albiol, D.; Stoumpos, C. C.: Raptopoulou, C. P.; Terzis, A.; Wernsdorfer, W.; Perlepes, S. P.; Christou, G. J. Am. Chem. Soc. 2005, 127, 15380.

5. (a) Hwang, S.-H.; Moorefield, C. N.; Wang. P.; Fronczek, F. R.; Courtney, B. H.; Newkome, G. R. Dalton Trans. 2006, 3518. (b) Hwang, S.-H.; Wang, P.; Moorefield, C. N.; Jung, J.-C.; Kim, J.Y.; Lee, S.-W.; Newkome, G. R. Macromol. Rapid Commun. 2006, 27, 1809 .

6. (a) Wong-Foy, A. G; Matzger, A. J.; Yaghi, O. M. J. Am. Chen. Soc. 2006, I28, 3494. (b) Rowsell, J. L. C.; Millward, A. R.; Park, K. S.; Yaghi, O. M. J. Am. Chem. Soc. 2004, 126, 5666. (c) Kubota, Y; Takata, M.; Malsuda, R,; Kitaura, R.; Kitagawa, S.; Kalo, K.; Sakata, M.; Kobayashi, T. C. Angew. Chem. Int. Ed. Entol. 2005, 44, 920. (d) Matsuda, R.; Kitaura, R.; Kitagawa, S.; Kubota, Y.; Belosludov, R. V.; Kobayashi, T. C.; Sakamoto, H.; Chiba. T.; Takata, M.; Kawazoe. Y.; Mita. Y. Nattre 2005, 436 . 238. (e) Millward, A. R.; Yaghi, O. M. J. Am. Chem. Soc. 2005, 127, 17998. (@) Uemura, K.; Kilagawa, S.; Fukui, K.; Sailo, K. J. Am. Chem. Soc. 2004, 126, 3817 .

7. (a) Yoshizawa, M.; Tamura, M.; Fujita, M. Science 2006, 3/2, 251. (b) Fiedler, D.; Leung, D. H.; Bergman, R. G.; Raymond, K. N. Ace. Chem. Res. 2005, 38, 351. (c) Fiedler, D.; Bergman, R. G.; Raymond, K. N. Angew. Chem. Int. Ed. Engl. 2004, 43, 6748. (d) Leung, D. H.; Fiedler, D.; Bergman, R. G.; Raymond, K. N. Angew. Chem. Int. Ed. Engl. 2004, 43, 963. (e) Seo, J. S.; Whang, D.; Lee, H.; Jun, S. I.; Oh, J.; Jeon, Y. J.; Kim, K. Nature 2000 , 404,982 .

8. (a) Kwak, B.; Rhee, H.; Park, S.; Lah, M. S. Inorg. Chem. 1998, 37, 3599. (b) Kwak, B.; Rhee, H.; Lah, M. S. Polyhedron 2000, 19, 1985. (c) John, R. P.; Lee, K.; Kim, B. J.; Suh, B. J.; Rhee, H.; Lah, M. S. Inorg. Chem. 2005, 44, 7109. (d) Moon, D.; Lee, K.; John, R. P.; Kim, G H.; Suh, B. J.; Lah, M. S. Inorg. Chem. 2006, 45. 7991. (e) Lin, S.; Liu, S.-X.; Huang, J.-Q.; Lin, C.-C. J. Chent. Soc, Dallon Trans. 2002, 1595. (f) Lin, S.; Liu, S.-X.; Chen, Z; Lin, B.-Z.; Gao, S. Inorg. Chem. 2004, 43, 2222. (g) Liu, S.-X.; Lin, S.-L.; Lin, B.-Z.; Lin, C.-C.; Huang, J.-Q. Angew. Chem. Int. Ed. Engl. 2001, 40, 1084 .

9. Song. J.; Moon, D.; Lah, M. S. Bull Korean Chem. Soc. 2002, 23, 708 .

10. John, R. P.; Lee, K.; Lah, M. S. Chem. Conmmtn. 2004, 2660.

11. SMART and SANT, Area Delector Soltware Package and SAX Area detector Integration Program; Bruker Analylical X-ray: Madison, WI, 1997.

12. SADABS, Area Detector Absorption Correction Program; Bruker Analytical X-ray: Madison, WI, 1997.

13. Sheldrick, G M. SHELITLPLUS, Crystal Structure Antabsis Package; Bruker Analytical X-ray: Madison, WI, 1997.

14. Platon program: Spek, A. L. Acta Cystallogr: Sect. A 1990, 46, 194. 